\title{
Who should define disease?
}

\author{
Fiona Godlee editor, BMJ
}

Last year an international panel of professional societies changed the definition of gestational diabetes. The blood glucose threshold for diagnosis was substantially lowered, more than doubling the number of women with the diagnosis. It will now encompass almost one in five pregnancies.

In his feature investigation this week (doi:10.1136/bmj.d2548), Ray Moynihan highlights this as just the latest example of how the definitions of common conditions are being broadened, so much so that by some estimates, almost the entire adult population is now classified as having at least one chronic disease.

Mental illness is an area of particular concern, he says, where controversy already bubbles around the definitions of attention deficit disorder, autism, and bipolar disorder. The revised diagnostic criteria produced by the American Psychiatric Association $(D S M-V)$ could, he warns, unleash a wave of what one psychiatrist calls "false positive epidemics."

And underlying the decisions to broaden disease definitions, he argues, are the conflicts of interest embedded among the decision makers. Of the panel members responsible for the current set of psychiatric definitions in $D S M-I V, 56 \%$ had financial ties to drug companies. The figure for $D S M-V$ is unchanged. In other fields the picture is similar. Half of the panel members who created the diagnosis of pre-hypertension in 2003 had extensive ties to industry.

As Moynihan says, excuses for the continuation of this state of affairs no longer wash. Those who argue that it's too hard to find experts who don't have industry ties are just not looking hard enough. Major decision making bodies-the Food and Drug Administration and the National Institutes of Health in America, and the UK's National Institute for Health and Clinical Excellence, among others-have adopted zero tolerance on experts with financial conflicts of interest. NIH has gone a step further, excluding those with a declared view on a question being considered. As one NIH senior manager says in the article, "intellectual conflicts of interest can be equally important."

Moynihan proposes going further still, again following the lead from the NIH. Its panels include a broad range of expertise and also, crucially, people representing the wider public interest. Since civil society has a major stake in decisions about where normality ends and disease begins, it should have a say in them as well.

Such panels will of course need good evidence on which to base their decisions, including evidence on the cost effectiveness of changing a diagnostic category. Therein lies the problem. As Moynihan points out, the type of evidence we need is in short supply: "the claims about the nature or extent of medical conditions are rarely exposed to the same rigorous systematic scrutiny as the studies of treatments for them."

I'm struck by the quote from Allen Frances, the psychiatrist who chaired the task force for DSM-IV. "New diagnoses are as dangerous as new drugs, he says. "We have remarkably casual procedures for defining the nature of conditions, yet they can lead to tens of millions being treated with drugs they may not need, and that may harm them."

So what should clinicians do, especially in their new extended role as commissioners of care? Be wary of new definitions of disease, question their provenance, make patients aware of the debate, demand greater transparency and tougher rules for decision makers, strive for independence yourself, and seek ways to encourage and reward it in others.

Cite this as: $B M J 2011 ; 342: \mathrm{d} 2974$ 